\title{
Set-Based Prediction of Pedestrians in Urban Environments Considering Formalized Traffic Rules
}

\author{
Markus Koschi ${ }^{1}$, Christian Pek ${ }^{1,2}$, Mona Beikirch ${ }^{1,2}$, and Matthias Althoff ${ }^{1}$
}

\begin{abstract}
Set-based predictions can ensure the safety of planned motions, since they provide a bounded region which includes all possible future states of nondeterministic models of other traffic participants. However, while autonomous vehicles are tested in urban environments, a set-based prediction tailored to pedestrians does not exist yet. This paper addresses this problem and presents an approach for set-based predictions of pedestrians using reachability analysis. We obtain tight overapproximations of pedestrians' reachable occupancy by incorporating the dynamics of pedestrians, contextual information, and traffic rules. In addition, since pedestrians often disregard traffic rules, our constraints automatically adapt so that such behaviors are included in the prediction. Using datasets of recorded pedestrians, we validate our proposed method and demonstrate its use for evasive maneuver planning of automated vehicles.
\end{abstract}

\section{INTRODUCTION}

\section{A. Motivation}

Automated vehicles may endanger other traffic participants in the event that they misjudge a traffic situation. In urban environments in particular, vulnerable road users such as pedestrians impose strict safety requirements. For example, if autonomous vehicles do not consider that an approaching pedestrian might try to cross the road at the last second (cf. Fig. 1), a fatal collision could be inevitable.

To prevent such situations at an early stage, the future motion of pedestrians needs to be accurately predicted [1], [2]. Current probabilistic approaches are limited when predicting all feasible and legal future motion, since they are not designed to enclose all behaviors given an uncertain pedestrian model. In contrast, set-based predictions guarantee that all planned motions are safe, even when traffic participants deviate from the most likely prediction [3]. Recently, a prediction approach using reachability analysis to account for any feasible future motion of other traffic participants in a set-based fashion was proposed [4]. However, a set-based prediction method for pedestrians considering both structured and unstructured environments does not yet exist, making it difficult to provide advanced safety systems which ensure the safety of vulnerable road users.

\footnotetext{
${ }^{1}$ Department of Informatics, Technical University of Munich, 85748 Garching, Germany.

${ }^{2}$ BMW Group, 85716 Unterschleissheim, Germany.

markus.koschi@tum.de, christian.pek@tum.de, mona.beikirch@tum.de, matthias.althoffetum.de

This work was partially supported by the BMW Group within the CAR@TUM project, the German Federal Ministry of Economics and Technology through the research initiative Ko-HAF, and the German Research Foundation (DFG) under grant number AL 1185/7-1.
}

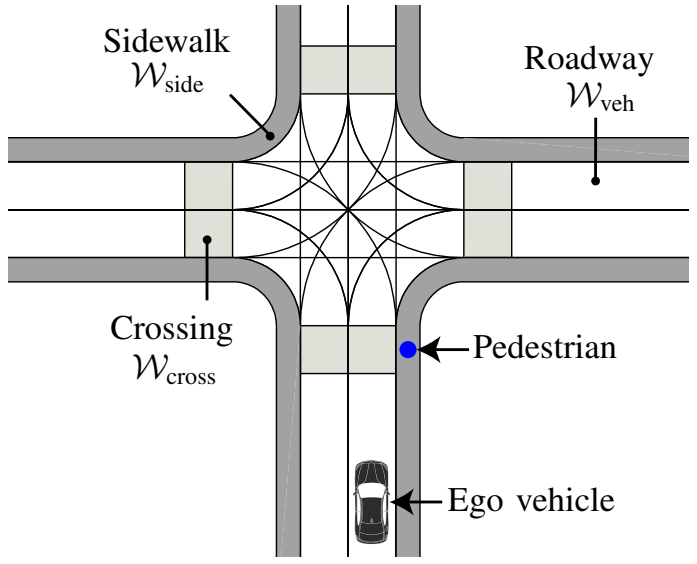

Fig. 1. The safety of the ego vehicle's planned motions can be guaranteed for given model assumptions by predicting all possible future behaviors of the pedestrian, which may include crossing the road even when traffic rules (e.g., a red light for the pedestrian) forbid such behavior.

\section{B. Related Work}

We review existing work on pedestrian prediction for automated vehicles in unknown environments categorized by whether they compute $a$ ) a single behavior, $b$ ) a probability distribution of multiple behaviors, or $c$ ) a bounded set of future behaviors. In order to apply such predictions, we require the current state of pedestrians from sensor data, which can be obtained as described in [5]-[7]; however, this process itself is beyond the scope of this work.

a) Single behavior: The probability of whether pedestrians intend to cross the roadway is computed in [8][12] using one or more of the following sources: motion information (previous path and current position), situation awareness (e.g., head pose), and contextual information (e.g., proximity to curb or intersection). Based on the predicted intention, the most likely behavior can be inferred, while other works directly compute a single trajectory [13], [14] or the time until the pedestrian will most likely cross [15].

b) Probability distribution: Predicting only a single behavior may suffice for short-term prediction; however, since many possible maneuvers exist, it is beneficial to compute a probability distribution of future behaviors by considering the possible goals of pedestrians [16]-[20].

c) Set of future behaviors: To verify that one does not collide with a pedestrian, a bounded set containing its possible future behaviors must be considered. While dynamicbased models have been used in [21]-[23], set-based models which integrate map-based information or traffic rules have not yet been developed, to the best of our knowledge. 


\section{Contribution}

This paper significantly extends previous work on setbased predictions [4], [23] by considering not only motorized traffic participants but also pedestrians, while exploiting traffic rules based on the given environment map. This extension will be available in the next version of our opensource prediction tool SPOT ${ }^{1}$ More specifically, our method is the first that can:

1) predict the feasible future motion of pedestrians in a formal and set-based manner,

2) obtain tight over-approximative occupancies by making use of formalized traffic rules and contextual information,

3) explicitly consider measurement uncertainties in the initial state of pedestrians, and

4) guarantee the safety of planned motions according to our assumptions.

The remainder of this paper is organized as follows. Sec. II introduces the required models and definitions, and Sec. III explains the set-based prediction of pedestrians. Sec. IV demonstrates our approach by using different datasets of recorded pedestrians and by evasive planning for autonomous vehicles. Finally, Sec. V] concludes the paper.

\section{PREliminaries}

\section{A. Road Model}

We model our environment in $\mathbb{R}^{2}$ using lanelets, which are atomic, interconnected, and drivable road segments [24]. Lanelets are defined using a left and right bound represented by a linearly interpolated list of points. As Fig. 11 shows, we distinguish two types of lanelets: vehicular lanelets (i.e., roadways) and pedestrian lanelets (i.e., sidewalks/pavements and crossings).

Definition 1 (Road networks)

We define the following types of road networks:

- The vehicular network is the union of all vehicular lanelets and is denoted by $\mathcal{W}_{\text {veh }} \subset \mathbb{R}^{2}$.

- The pedestrian network $\mathcal{W}_{\text {ped }} \subset \mathbb{R}^{2}$ is the union of all pedestrian lanelets, i.e., sidewalks $\mathcal{W}_{\text {side }}$ and crossings $\mathcal{W}_{\text {cross. }}$ We use $\mathcal{W}_{\text {cross }}^{\text {prio }}(t)$ to denote the crossings a pedestrian is allowed to cross at time $t$ (cf. Sec. IIIB).

- The forbidden network $\mathcal{W}_{\text {forbid }}:=\mathcal{W}_{\text {veh }} \cap \mathcal{W}_{\text {ped }}^{\complement}$ is the part of $\mathcal{W}_{\text {veh }}$ pedestrians are not allowed to enter (where $\mathcal{W}_{\text {ped }}^{\complement}$ denotes the complement of $\mathcal{W}_{\text {ped }}$, cf. Fig. 4). The boundary of $\mathcal{W}_{\text {forbid }}$ is denoted by $\delta \mathcal{W}_{\text {forbid. }}$

Let a disk, i.e., a circular area, with center $\left[c_{x}, c_{y}\right]^{T}$ and radius $r$ be denoted as $\mathcal{C}\left(\left[c_{x}, c_{y}\right]^{T}, r\right):=\left\{\left[s_{x}, s_{y}\right]^{T} \mid\left(s_{x}-\right.\right.$ $\left.\left.c_{x}\right)^{2}+\left(s_{y}-c_{y}\right)^{2} \leq r^{2}\right\}$. If $c_{x}=c_{y}=0$, we just write $\mathcal{C}(r)$. The following predicates are defined using first-order logic to argue about the position of pedestrians:

\footnotetext{
${ }^{1}$ Available at spot.in.tum.de
}

Definition 2 (Not intruding $\mathcal{W}_{\text {forbid }}$ )

The predicate notInWf $\left(\mathcal{X}_{s}, r\right)$ evaluates to true if all points $\left[s_{x}, s_{y}\right]^{T} \in \mathcal{X}_{s} \subset \mathbb{R}^{2}$ intrude the vehicular network by at most the distance $r$ :

$$
\operatorname{notInWf}\left(\mathcal{X}_{s}, r\right) \Leftrightarrow\left(\mathcal{W}_{\text {forbid }} \ominus \mathcal{C}(r)\right) \cap \mathcal{X}_{s}=\emptyset,
$$

where $\ominus$ denotes the Minkowski difference defined for sets $\mathcal{A}$ and $\mathcal{B}$ as $\mathcal{A} \ominus \mathcal{B}:=\left(\mathcal{A}^{\complement} \oplus \mathcal{B}\right)^{\complement}$ using the Minkowski addition $(\mathcal{A} \oplus \mathcal{B}:=\{a+b \mid a \in \mathcal{A}, b \in \mathcal{B}\})$.

Definition 3 (Conforming to crossing priority)

The predicate confPrio $\left(\mathcal{X}_{s}, t\right)$ evaluates to true if none of the points $\left[s_{x}, s_{y}\right]^{T} \in \mathcal{X}_{s}$ are located in a forbidden crossing at time $t$ :

$$
\operatorname{confPrio}\left(\mathcal{X}_{s}, t\right) \Leftrightarrow \mathcal{W}_{\text {cross }} \cap \mathcal{W}_{\text {cross }}^{\text {prio }}(t)^{\complement} \cap \mathcal{X}_{s}=\emptyset .
$$

\section{B. Reachable Set of Pedestrians}

The motion of a pedestrian can be described by the differential equation

$$
\dot{x}(t)=f(x(t), u(t)),
$$

where $x \in \mathbb{R}^{n}$ is the state, $u \in \mathbb{R}^{m}$ is the input, and $t$ is the time. The possible states and inputs are bounded by the sets $\mathcal{X}$ and $\mathcal{U}$, respectively. We denote the initial time by $t_{0}$, the final time by $t_{f}>t_{0}$, an input trajectory by $u(\cdot)$, and a possible solution of (1) at time $t$ by $\chi\left(t, x\left(t_{0}\right), u(\cdot)\right)$.

Definition 4 (Reachable set)

The reachable set $\mathcal{R} \subseteq \mathcal{X}$ of (1) is the set of states which are reachable at time $t$ from an initial set $\mathcal{X}^{0} \subseteq \mathcal{X}$ at time $t_{0}$ and subject to the set of inputs $\mathcal{U}$ :

$$
\begin{aligned}
\mathcal{R}(t)= & \left\{\chi\left(t, x\left(t_{0}\right), u(\cdot)\right) \mid x\left(t_{0}\right) \in \mathcal{X}^{0},\right. \\
& \left.\forall t^{\star} \in\left[t_{0}, t\right]: \chi\left(t^{\star}, x\left(t_{0}\right), u(\cdot)\right) \in \mathcal{X}, u\left(t^{\star}\right) \in \mathcal{U}\right\} .
\end{aligned}
$$

Our state vector is $x=\left[s_{x}, s_{y}, v_{x}, v_{y}\right]^{T}$, where $s_{x}$ and $s_{y}$ denote the position, $v_{x}$ and $v_{y}$ the velocity, each in $x$ - and $y$-direction, respectively. We define the occupancy of a state as:

\section{Definition 5 (Occupancy of a state)}

The operator $\operatorname{occ}(x)$ returns the set of points in the twodimensional Cartesian space occupied by the pedestrian in state $x$ due to its circular dimensions with radius $r_{p e d}$ :

$$
\operatorname{occ}(x):=\left\{P x \oplus \mathcal{C}\left(r_{\text {ped }}\right)\right\}
$$

where $P$ is the projection matrix $P=[I \mathbf{0}] \in \mathbb{R}^{2 \times 4}, I$ the identity matrix, and $\mathbf{0}$ a matrix of zeros, both with proper dimensions. Given a set of states $\mathcal{X}$, the operator is defined as $\operatorname{occ}(\mathcal{X}):=\{\operatorname{occ}(x) \mid x \in \mathcal{X}\}$.

To obtain the future occupancy of pedestrians efficiently, we over-approximate their reachable occupancy:

Definition 6 (Ore-approximative occupancy set) Based on Def. 4 and Def. 5 the occupancy set $\mathcal{O}(t)$ overapproximates the set of occupied points which are reachable by the pedestrian: $\mathcal{O}(t) \supseteq \operatorname{occ}(\mathcal{R}(t))$. 


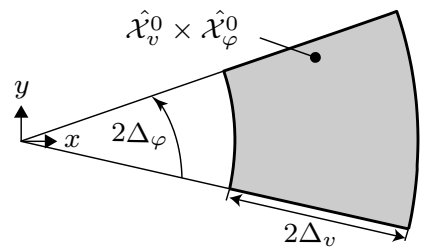

Fig. 2. The set of initial velocities, i.e., $\hat{\mathcal{X}}_{v}^{0} \times \hat{\mathcal{X}}_{\varphi}^{0}$, is modeled by an annulus sector, i.e., a circular ring sector.

Since an occupancy $\mathcal{O}(t)$ can be non-convex, we represent it by a polygon, and since a collision check with the intended trajectory of the ego vehicle requires an infinite number of points in time to be checked, we compute occupancies for consecutive time intervals $\tau_{k}=\left[t_{k}, t_{k+1}\right] \subseteq\left[t_{0}, t_{f}\right]$ with time step size $\Delta t=t_{k+1}-t_{k}$.

The initial set $\mathcal{X}^{0}$ in Def. 4 contains measurement uncertainties. Using a polar coordinate system to describe $v_{x}$ and $v_{y}$ by the radius $v$ and the polar angle $\varphi$, we introduce the following initial sets:

$$
\begin{aligned}
\hat{\mathcal{X}_{s}^{0}} & :=\mathcal{C}\left(s_{0}, \Delta_{s}\right), \\
\hat{\mathcal{X}_{v}^{0}} & :=\left[v_{0}-\Delta_{v}, v_{0}+\Delta_{v}\right], \\
\hat{\mathcal{X}_{\varphi}^{0}} & :=\left[\varphi_{0}-\Delta_{\varphi}, \varphi_{0}+\Delta_{\varphi}\right],
\end{aligned}
$$

where $s_{0}:=\left[s_{x_{0}}, s_{y_{0}}\right]^{T}$, and $\Delta_{s}, \Delta_{v}$, and $\Delta_{\varphi}$ denote the measurement uncertainty of the corresponding variable. As Fig. 2 shows, the set of initial velocities is bounded by an annulus sector. The initial set $\hat{\mathcal{X}}^{0}$ is constructed by the Cartesian product of the partial initial sets $\hat{\mathcal{X}}^{0}:=\hat{\mathcal{X}}_{s}^{0} \times \hat{\mathcal{X}}_{v}^{0} \times$ $\hat{\mathcal{X}}_{\varphi}^{0}$; the set $\hat{\mathcal{X}}^{0}$ in Cartesian coordinates is denoted by $\mathcal{X}^{0}$. The initial occupancy is $\mathcal{O}^{0}:=\operatorname{occ}\left(\mathcal{X}^{0}\right)$, which accounts for uncertainties in the pedestrian's dimensions by choosing $r_{\text {ped }}$ as the maximum of the measured radii.

\section{Prediction of Pedestrians}

To efficiently compute a tight over-approximative occupancy of (1), we use two types of occupancies: 1) the occupancy $\mathcal{O}_{\text {dyn }}(t)$ considering the dynamics of the pedestrian and 2) the occupancy $\mathcal{O}_{\text {rule }}(t)$ considering possible states according to traffic rules, as described in Sec. III-A and Sec. III-B respectively. Then, the over-approximative occupancy is the intersection of both over-approximations:

$$
\forall \tau_{k} \subseteq\left[t_{0}, t_{f}\right]: \mathcal{O}\left(\tau_{k}\right)=\mathcal{O}_{\text {dyn }}\left(\tau_{k}\right) \cap \mathcal{O}_{\text {rule }}\left(\tau_{k}\right)
$$

\section{A. Dynamic-Based Occupancy}

We use a kinematic model for pedestrians:

\section{Definition 7 (Dynamic model of pedestrians)}

The dynamics of a pedestrian are described by a velocityand acceleration-bounded point mass:

$$
\begin{gathered}
\ddot{s}_{x}=u_{x}, \ddot{s}_{y}=u_{y}, \\
\sqrt{\left|u_{x}\right|^{2}+\left|u_{y}\right|^{2}} \leq a_{\max }, \\
\sqrt{\left|v_{x}\right|^{2}+\left|v_{y}\right|^{2}} \leq v_{\max },
\end{gathered}
$$

where $u_{x}$ and $u_{y}$ denote the acceleration input in the $x$ and $y$-direction, respectively, $a_{\max }$ the maximum allowed acceleration, and $v_{\max }$ the maximum allowed velocity.

To efficiently obtain the occupancy, we do not directly perform reachability analysis on (6), but use the approach proposed by [23]: We separately compute an accelerationconstrained occupancy $\mathcal{O}_{\text {acc }}(t)$ considering only the constraint $6 \mathrm{~b}$ and a velocity-constrained occupancy $\mathcal{O}_{\text {vel }}(t)$ considering only the constraint 6c), as explained in Sec. IIIA.1 and III-A.2 respectively.

\section{1) Acceleration-constrained occupancy:}

Proposition 1 (Reachable positions $\mathcal{R}_{\text {acc }}^{\text {pos }}(t)$ for point in time) The reachable positions $\mathcal{R}_{\text {acc }}^{\text {pos }}(t)$ of $6 \mathrm{a}$ with $6 \mathrm{~b}$ are

$$
\mathcal{R}_{\text {acc }}^{\text {pos }}(t)=\Gamma_{\text {hom }}(t) \mathcal{X}^{0} \oplus \Gamma_{\text {inp }}(t) \mathcal{U}
$$

where

$$
\Gamma_{\text {hom }}(t)=\left[\begin{array}{llll}
1 & 0 & t & 0 \\
0 & 1 & 0 & t
\end{array}\right], \quad \Gamma_{\text {inp }}(t)=\frac{1}{2} t^{2}\left[\begin{array}{ll}
1 & 0 \\
0 & 1
\end{array}\right] .
$$

Proof: Let us first write (6a) in state-space form:

$$
\underbrace{\left[\begin{array}{c}
\dot{s}_{x} \\
\dot{s}_{y} \\
\dot{v}_{x} \\
\dot{v}_{y}
\end{array}\right]}_{\dot{x}}=\underbrace{\left[\begin{array}{cccc}
0 & 0 & 1 & 0 \\
0 & 0 & 0 & 1 \\
0 & 0 & 0 & 0 \\
0 & 0 & 0 & 0
\end{array}\right]}_{A} \underbrace{\left[\begin{array}{c}
s_{x} \\
s_{y} \\
v_{x} \\
v_{y}
\end{array}\right]}_{x}+\underbrace{\left[\begin{array}{cc}
0 & 0 \\
0 & 0 \\
1 & 0 \\
0 & 1
\end{array}\right]}_{B} \underbrace{\left[\begin{array}{l}
u_{x} \\
u_{y}
\end{array}\right]}_{u} .
$$

In general, the exact reachable set of linear systems cannot be computed, except for when $A$ is nilpotent or the eigenvalues are purely real or imaginary [25]. Since $A$ is nilpotent $\left(A^{2}\right.$ is a matrix of zeros), we can compute the exact reachable set as presented in [26, Sec. 3.2]:

$$
\begin{aligned}
& \mathcal{R}_{\text {acc }}(t)=e^{A t} \mathcal{X}^{0} \oplus \bigoplus_{i=0}^{\infty} \frac{A^{i} t^{i+1}}{(i+1) !} B \mathcal{U} \\
& \stackrel{\text { nilpotence }}{=}(I+A t) \mathcal{X}^{0} \oplus(B \mathcal{U} t) \oplus \frac{1}{2} A t^{2} B \mathcal{U} .
\end{aligned}
$$

Since we are only interested in the reachable set of the positions, we multiply the above solution with the projection

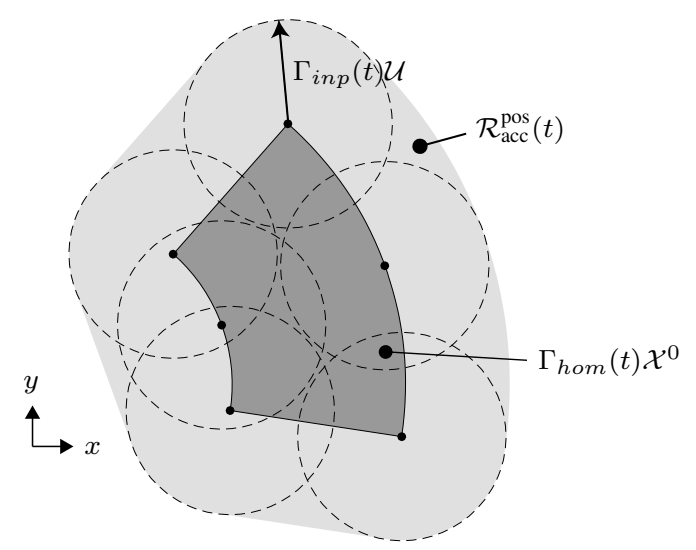

Fig. 3. The reachable positions of the acceleration-constrained mode $\mathcal{R}_{\text {acc }}^{\mathrm{pos}}(t)$ are bounded by the Minkowski addition of $\Gamma_{h o m}(t) \mathcal{X}^{0}$ with $\Gamma_{\text {inp }}(t) \mathcal{U}$ (cf. Prop. 11. 
TABLE I

DEFINITION OF THE OCCUPANCIES BASED ON FORMALIZED TRAFFIC RULES, WHICH ARE COMPUTED DEPENDING ON THEIR BOOLEAN VARIABLE.

\begin{tabular}{|c|c|c|c|c|c|c|c|c|}
\hline Constraint & Boolean & Description & Traffic rule [29] & \multicolumn{5}{|c|}{ Occupancy } \\
\hline$C_{\text {prio }}$ & $b_{\text {prio }}$ & $\begin{array}{l}\text { Within } \mathcal{W}_{\text {cross, }} \text { pedestrians are allowed to } \\
\text { cross if they have priority over vehicular } \\
\text { traffic, i.e., at pedestrian crossings, at inter- } \\
\text { sections when pedestrians have green traffic } \\
\text { lights, and at intersections without traffic } \\
\text { lights when vehicles take a turn }{ }^{2}\end{array}$ & $20 \S 6(b), 21 \S 2$ & $\mathcal{O}_{\text {prio }}(t)=$ & $= \begin{cases}\mathcal{W}_{\text {cross }}^{\text {prio }}(t), & b_{\text {pri }} \\
\mathcal{W}_{\text {cross }}, & \neg b\end{cases}$ & $\begin{array}{l}\text { io } \\
\text { prio. }\end{array}$ & & \\
\hline$C_{\text {stop }}$ & $b_{\text {stop }}$ & $\begin{array}{l}\text { When (carelessly) stepping on the road- } \\
\text { way outside } \mathcal{W}_{\text {cross }} \text { (i.e., onto } \mathcal{W}_{\text {forbid }} \text { ), the } \\
\text { pedestrian immediately slows down with } \\
a_{\text {stop to come to a stop as soon as possible }} \\
\text { in order to not impede vehicular traffic. }\end{array}$ & $20 \S 6(a, c)$ & $\mathcal{O}_{\text {stop }}=$ & $\begin{array}{l}\emptyset \\
\mathcal{C}\left(s_{0}, r_{\text {stop }}+r_{\text {ped }}\right) \\
\mathcal{C}\left(p_{\mathcal{W}}\left(s_{0}\right), r_{\text {stop }}+\right.\end{array}$ & $\begin{array}{l} \\
\left.+r_{\mathrm{ped}}\right)\end{array}$ & $\begin{array}{l}b_{\text {stop }}, \\
\neg b_{\text {stop }} \\
\neg b_{\text {stop }}\end{array}$ & $\begin{array}{l}\wedge \operatorname{not} \operatorname{InWf}\left(\mathcal{O}^{0}, 0\right) \\
\wedge \neg \operatorname{notInWf}\left(\mathcal{O}^{0}, 0\right)\end{array}$ \\
\hline$C_{\text {perp }}$ & $b_{\text {perp }}$ & $\begin{array}{l}\text { Crossing the roadway outside } \mathcal{W}_{\text {cross }} \text { is } \\
\text { not allowed. If crossing nevertheless, the } \\
\text { shortest path of width } \xi_{\text {perp }} \text {, which is per- } \\
\text { pendicular to the driving direction, must be } \\
\text { chosen. }\end{array}$ & $20 \S 6(\mathrm{c}, \mathrm{d})$ & $\mathcal{O}_{\text {perp }}=$ & $\emptyset$ & $\begin{array}{l}b_{\text {perp }}, \\
\neg b_{\text {perp }} .\end{array}$ & & \\
\hline$C_{\text {slack }}$ & $b_{\text {slack }}$ & $\begin{array}{l}\text { Walking on the roadway is not allowed; } \\
\mathcal{W}_{\text {forbid }} \text { may be entered by the margin } \xi_{\text {slack }} \\
\text { only if no usable sidewalk is provided. }\end{array}$ & $\begin{array}{c}20 \S 2(a), 20 \S 3, \\
20 \S 4\end{array}$ & $\mathcal{O}_{\text {slack }}=$ & $\left\{\begin{array}{l}\emptyset \\
\left(\mathcal{W}_{\text {forbid }}^{\complement} \oplus \mathcal{C}\left(\xi_{\text {slac }}\right.\right.\end{array}\right.$ & $\mathrm{ck})) \cap$ & forbid, & $\begin{array}{l}b_{\text {slack }}, \\
\neg b_{\text {slack }} .\end{array}$ \\
\hline
\end{tabular}

matrix $P$ (cf. Fig. 3):

$$
\mathcal{R}_{\mathrm{acc}}^{\mathrm{pos}}(t)=P \mathcal{R}_{\mathrm{acc}}(t)=\underbrace{\left[\begin{array}{cccc}
1 & 0 & t & 0 \\
0 & 1 & 0 & t
\end{array}\right]}_{\Gamma_{\mathrm{hom}}} \mathcal{X}^{0} \oplus \underbrace{\frac{1}{2} t^{2}\left[\begin{array}{ll}
1 & 0 \\
0 & 1
\end{array}\right]}_{\Gamma_{\mathrm{inp}}} \mathcal{U} \text {. }
$$

Next, we consider the reachable set for time intervals.

Proposition 2 (Reachable positions $\mathcal{R}_{\text {acc }}^{\text {pos }}\left(\tau_{k}\right)$ for time interval) The reachable positions of the acceleration-bounded model for a time interval $\tau_{k}=\left[t_{k}, t_{k+1}\right]$ are

$$
\begin{aligned}
\mathcal{R}_{\text {acc }}^{\text {pos }}\left(\tau_{k}\right)= & \operatorname{conv}\left(\Gamma_{\text {hom }}\left(t_{k}\right) \mathcal{X}^{0}, \Gamma_{\text {hom }}\left(t_{k+1}\right) \mathcal{X}^{0}\right) \\
& \oplus \Gamma_{\text {inp }}\left(t_{k+1}\right) \mathcal{U},
\end{aligned}
$$

where $\operatorname{conv}(\mathcal{A}, \mathcal{B})$ returns the convex hull of the sets $\mathcal{A}$ and $\mathcal{B}$.

Proof: The proof follows directly from [27, Alg. 1], where the matrix $\mathcal{F}$ in that algorithm is a matrix of zeros due to the nilpotence of $A$.

Finally, the acceleration-constrained occupancy for a single point in time is $\mathcal{O}_{\text {acc }}(t)=\operatorname{occ}\left(\mathcal{R}_{\text {acc }}^{\text {pos }}(t)\right)$ and $\mathcal{O}_{\text {acc }}\left(\tau_{k}\right)=$ $\operatorname{occ}\left(\mathcal{R}_{\text {acc }}^{\text {pos }}\left(\tau_{k}\right)\right)$ for a time interval.

2) Velocity-constrained occupancy: Up until now, we have ignored the maximum velocity constraint in (6c). Let us first determine the earliest point in time when the maximum velocity is reached:

$v_{\max }=v_{0}+\Delta_{v}+a_{\max } t_{\mathrm{v}_{\max }} \Leftrightarrow t_{\mathrm{v}_{\max }}=\frac{v_{\max }-\left(v_{0}+\Delta_{v}\right)}{a_{\max }}$.

When starting at the origin with the maximum velocity in all directions, the reachable set is a disk centered at the origin

\footnotetext{
${ }^{2}$ In order to automatically consider that pedestrians have priority over turning vehicles at intersections, it is necessary to compute this occupancy depending on the intended motion of the ego vehicle.
}

with radius $v_{\max }\left(t-t_{\mathrm{v}_{\max }}\right)$. Thus, an over-approximation of the reachable positions considering the velocity constraint for $t>t_{\mathrm{v}_{\max }}$ is

$$
\mathcal{O}_{\text {vel }}(t)=\mathcal{O}_{\text {acc }}\left(t_{\mathrm{v}_{\max }}\right) \oplus \mathcal{C}\left(v_{\max }\left(t-t_{\mathrm{v}_{\max }}\right)\right) .
$$

Due to the monotonic growth of $\mathcal{C}\left(v_{\max }\left(t-t_{\mathrm{v}_{\max }}\right)\right)$, it follows that $\mathcal{C}\left(v_{\max }\left(t_{k+1}-t_{\mathrm{v}_{\max }}\right)\right) \supseteq \mathcal{C}\left(v_{\max }\left(t_{k}-t_{\mathrm{v}_{\max }}\right)\right)$, and thus

$$
\mathcal{O}_{\text {vel }}\left(\tau_{k}\right)=\mathcal{O}_{\text {acc }}\left(t_{\mathrm{v}_{\max }}\right) \oplus \mathcal{C}\left(v_{\max }\left(t_{k+1}-t_{\mathrm{v}_{\max }}\right)\right) .
$$

Since $\mathcal{O}_{\text {vel }}(t)$ is not required for $t \leq t_{\mathrm{v}_{\max }}$, the overall dynamic-based occupancy is

$$
\mathcal{O}_{\text {dyn }}\left(\tau_{k}\right)= \begin{cases}\mathcal{O}_{\mathrm{acc}}\left(\tau_{k}\right), & t_{k} \leq t_{\mathrm{v}_{\max }} \\ \mathcal{O}_{\mathrm{acc}}\left(\tau_{k}\right) \cap \mathcal{O}_{\mathrm{vel}}\left(\tau_{k}\right), & t_{k}>t_{\mathrm{v}_{\max }}\end{cases}
$$

\section{B. Rule-Based Occupancy}

We incorporate formalized traffic rules into our prediction [28]. As a legal source, we use the Vienna Convention on Road Traffic [29]. We assume that pedestrians adhere to traffic rules and do not obstruct vehicular traffic [29, $7 \S 1]$. However, if pedestrians violate rules, we have to take necessary precautions to avoid endangering pedestrians [29, $21 \S 1]$.

Pedestrians are generally not allowed to leave the pedestrian network and enter the roadway [29, 20§2]. Since this rule has different cases of violations, we deduce four atomic constraints described in Tab. If these constraints each have a Boolean variable, denoted by $b$, which allows us to enable and disable this constraint individually.

The occupancies resulting from these constraints are illustrated in Fig. 4 and are computed as presented in Tab. I where we use the following variables: The distance required 
TABLE II

THE CONSTRAINT MANAGEMENT DEACTIVATES OR ADAPTS THE CONSTRAINTS IF UNDERLYING ASSUMPTIONS ARE VIOLATED.

\begin{tabular}{lll}
\hline Constraint & Parameter with default values & Condition adapting the constraint variable \\
\hline$C_{\text {prio }}$ & - & $b_{\text {prio }} \Leftrightarrow \operatorname{confPrio}\left(\mathcal{O}^{0}, t_{0}\right) \wedge b_{\text {perp }}$ \\
$C_{\text {stop }}$ & $a_{\text {stop }}=0.6 \mathrm{~m} / \mathrm{s}^{2}$ & $b_{\text {stop }} \Leftrightarrow \forall t \in\left[t_{0}, t_{f}\right]:\left(\mathcal{O}_{\text {dyn }}(t) \cap \mathcal{O}_{\text {rule }}(t)\right) \ominus \mathcal{C}\left(r_{\text {ped }}\right) \neq \emptyset$ \\
$C_{\text {perp }}$ & $\xi_{\text {perp }}=2.0 \mathrm{~m}$ & $b_{\text {perp }} \Leftrightarrow \operatorname{notInWf}\left(\mathcal{O}^{0}, \max \left(\xi_{\text {slack }}, r_{\text {stop }}+r_{\text {ped }}\right)\right) \wedge\left(b_{\text {stop }} \vee \forall t \in\left[t_{0}, t_{f}\right]:\right.$ \\
& & $\left.\left(\mathcal{O}_{\text {dyn }}(t) \cap \mathcal{O}_{\text {rule }}(t)\right) \ominus \mathcal{C}\left(r_{\text {ped }}\right) \neq \emptyset\right)$ \\
$C_{\text {slack }}$ & $\xi_{\text {slack }}=1.0 \mathrm{~m}$ & $b_{\text {slack }} \Leftrightarrow \operatorname{notInWf}\left(\mathcal{O}^{0}, 0\right)$ \\
$C_{a_{\text {max }}}$ & $a_{\text {max }}=0.6 \mathrm{~m} / \mathrm{s}^{2}, \Delta_{a_{\text {max }}}=0.05 \mathrm{~m} / \mathrm{s}^{2}$ & $a_{\text {max }} \leftarrow \max \left(a_{\max }, a_{0}+\Delta_{a}+\Delta_{a_{\text {max }}}\right)$ \\
$C_{v_{\text {max }}}$ & $v_{\text {max }}=2 \mathrm{~m} / \mathrm{s}, \Delta_{v_{\text {max }}}=0.1 \mathrm{~m} / \mathrm{s}$ & $v_{\max } \leftarrow \max \left(v_{\max }, v_{0}+\Delta_{v}+\Delta_{v_{\text {max }}}\right)$ \\
\hline
\end{tabular}

for the pedestrian to stop with deceleration $a_{\text {stop }}$ from its current velocity is $r_{\text {stop }}:=\frac{1}{2 a_{\text {stop }}}\left(v_{0}+\Delta_{v}\right)^{2}+\Delta_{s}$. The point on $\delta \mathcal{W}_{\text {forbid }}$ closest to the center of $\mathcal{O}^{0}$ is $p_{\mathcal{W}}\left(s_{0}\right):=$ $\operatorname{argmin}\left\|p-s_{0}\right\|_{2}$ (cf. Fig. 4), and the unit vector at $p_{\mathcal{W}}\left(s_{0}\right)$ $p \in \delta \mathcal{W}_{\text {forbid }}$ tangential to $\delta \mathcal{W}_{\text {forbid }}$ is denoted by $t_{\mathcal{W}}\left(s_{0}\right)$. We choose $s_{0}$ as the center of $\mathcal{O}_{\text {stop }}$, since we assume that pedestrians immediately slow down to avoid entering the road (i.e., entering $\mathcal{W}_{\text {forbid }}$ ); however, for a pedestrian already located in $\mathcal{W}_{\text {forbid }}$, we assume that the pedestrian had started slowing down when entering $\mathcal{W}_{\text {forbid }}$, and thus choose $p_{\mathcal{W}}\left(s_{0}\right)$ as the center of $\mathcal{O}_{\text {stop }}$ (cf. Tab. I). For $\mathcal{O}_{\text {perp }}$, we compute the area perpendicular to the roadway by

$\mathcal{W}_{\text {perp }}:=\left\{\left[\begin{array}{l}s_{x} \\ s_{y}\end{array}\right]\left\|t_{\mathcal{W}}\left(s_{0}\right)^{T}\left(\left[\begin{array}{l}s_{x} \\ s_{y}\end{array}\right]-p_{\mathcal{W}}\left(s_{0}\right)\right)\right\| \leq \frac{\xi_{\text {perp }}}{2}\right\}$,

where the parameter $\xi_{\text {perp }}$ describes the width of this corridor (cf. Fig. 4).

Finally, we define the rule-based occupancy $\mathcal{O}_{\text {rule }}(t)$ as the area of the whole vehicular and pedestrian network respecting the constraints of Tab. Ir

Definition 8 (Rule-based occupancy)

Using the partial occupancies from Tab. I the rule-based occupancy of a pedestrian is

$$
\mathcal{O}_{\text {rule }}(t)=\mathcal{W}_{\text {side }} \cup \mathcal{O}_{\text {prio }}(t) \cup \mathcal{O}_{\text {stop }} \cup \mathcal{O}_{\text {perp }} \cup \mathcal{O}_{\text {slack }}
$$

Note that all partial occupancies of $\mathcal{O}_{\text {rule }}(t)$, except $\mathcal{O}_{\text {prio }}(t)$,

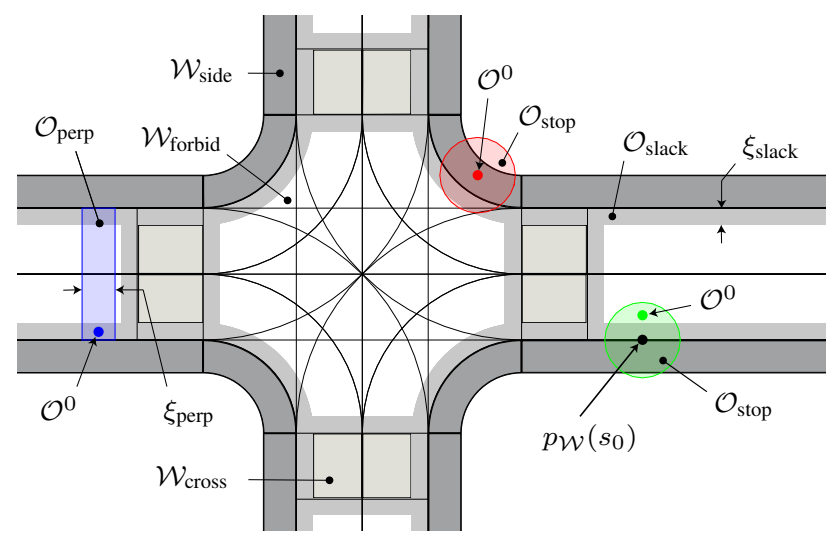

Fig. 4. Visualization of the occupancies based on formalized traffic rules of Tab. [] are constant over the prediction horizon, and $\mathcal{W}_{\text {side }}, \mathcal{W}_{\text {cross }}$, and $\mathcal{O}_{\text {slack }}$ can be precomputed offline for given road networks.

\section{Constraint Management}

The prediction of each pedestrian is based on traffic rules, which are represented by the constraints introduced in Tab. I] The Boolean variables are initialized with $b=$ true and then set according to the conditions listed in the last column of Tab. III Thus, constraints are automatically deactivated as soon as assumptions on the traffic rules are violated. Let us explain the constraint management for $C_{\text {stop }}$ and $C_{\text {perp }}$ in more detail. For $C_{\text {stop }}$, pedestrians are anticipated to step on the roadway if they cannot stop before; in this case, $b_{\text {stop }}=$ false. The condition of $C_{\text {perp }}$ further anticipates that the pedestrian crosses the road if stopping within $\mathcal{O}_{\text {stop }}$ is not possible or if $\mathcal{W}_{\text {forbid }}$ is intruded by more than the maximum of $\xi_{\text {slack }}$ and $r_{\text {stop }}+r_{\text {ped }}$; in these cases, $b_{\text {perp }}=$ false.

Note that the proposed conditions for the constraints are deduced from traffic rules, but may be adapted to obtain more or less conservative behavior, e.g., a sophisticated intention prediction may directly set $b_{\text {perp }}$ and $b_{\text {slack }}$ to false (and increase $\xi_{\text {perp }}$ and $\xi_{\text {slack }}$ ) for a child playing at the side of the road. Thus, our approach offers the possibility of deactivating constraints based on the specifications of users while still remaining formally valid.

For the dynamic-based occupancy, the constraints (6b) and (6c) are not deactivated if we measure higher values; instead, their maximum allowed values are adjusted as presented in the last two rows of Tab. II where the parameters $\Delta_{a_{\max }}$ and $\Delta_{v_{\max }}$ are thresholds to anticipate that the measured values might be exceeded and thus, the updated maximum values will not be directly violated again.

\section{Evaluation With REAL-World Data}

We evaluate our approach using recorded data with measurement noise obtained from a moving vehicle [30]. Fig. 5 shows the view from the front camera of the autonomous vehicle and three tracked pedestrians crossing the street. The default values for the constraints in our prediction are listed in Tab. II For the dynamic-based occupancy, the maximum acceleration and velocity must be parametrized. As in [23], we use as the default $a_{\max }=0.6 \mathrm{~m} / \mathrm{s}^{2}$ (based on a labeled video source [31]) and $v_{\max }=2.0 \mathrm{~m} / \mathrm{s}$ (which 


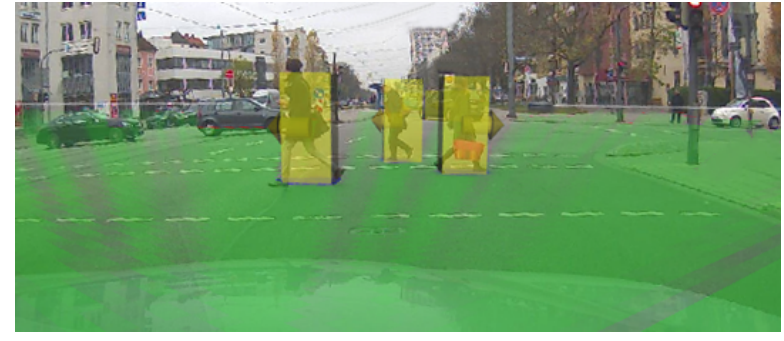

Fig. 5. View from the front camera of the autonomous vehicle approaching three pedestrians crossing the street. The recorded data is provided by [30].

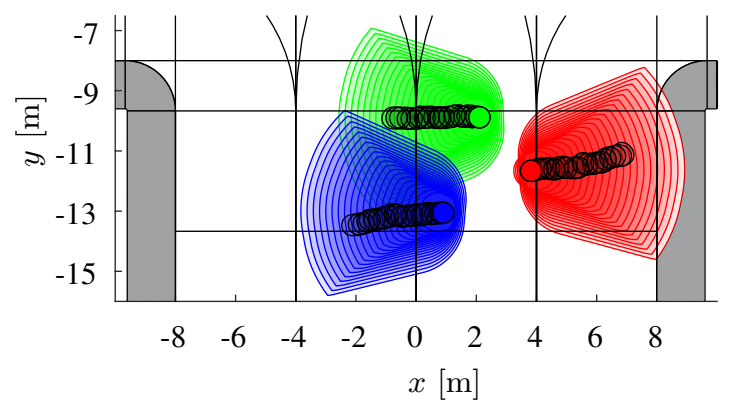

(a) $\mathcal{O}_{\text {dyn }}\left(\tau_{k}\right), k=0, \ldots, 19$.

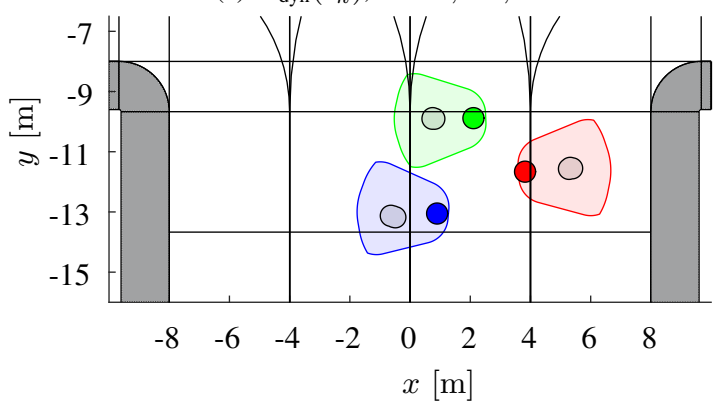

(b) $\mathcal{O}_{\text {dyn }}\left(\tau_{9}\right)$.

Fig. 6. The predicted dynamic-based occupancy contains the recorded occupancy of the three pedestrians at the crossing of Fig. 5

is the transition speed between walking and running and is suggested by [32]). One can also choose different values, e.g., from extensive physiological experiments on walking, running, and stopping [33]. Our results have been obtained using MATLAB 2016a on a machine with a $2.6 \mathrm{GHz}$ Intel Core i7 processor with $20 \mathrm{~GB} 1600 \mathrm{MHz}$ DDR3 memory.

\section{A. Conformance of Dynamic-Based Occupancy}

We validate our dynamic-based occupancy by checking whether our model over-approximates the real behavior of walking-only pedestrians, using $\Delta t=0.1 \mathrm{~s}, t_{f}-t_{0}=$ $2.0 \mathrm{~s}$, and $r_{\text {ped }}=0.35 \mathrm{~m}$. From [30], we predict 11 pedestrians for a total of $7008 \mathrm{~s}$ with $\Delta_{s} \in[0.19 \mathrm{~m}, 0.96 \mathrm{~m}]$, $\Delta_{v} \in[0.21 \mathrm{~m} / \mathrm{s}, 1.5 \mathrm{~m} / \mathrm{s}]$, and $\Delta_{\varphi} \in[0.21 \mathrm{rad}, 0.88 \mathrm{rad}]$. We achieve a coverage of $100 \%$, since all recorded occupancies, i.e., ground-truth trajectories without uncertainty enlarged by $r_{\text {ped }}$, were fully contained within the predicted $\mathcal{O}_{\text {dyn }}\left(\tau_{k}\right)$. As an example, Fig. 6a depicts our result of the three pedestrians from Fig. 5 A snapshot in Fig. 6b for $\tau_{9}=[0.9 \mathrm{~s}, 1.0 \mathrm{~s}]$ shows that our set-based prediction is not unreasonably conservative. Note that our prediction remains over-approximative for longer time horizons (tested for up to $5.0 \mathrm{~s})$.

The computation time for each pedestrian was $23 \mathrm{~ms}$; however, since the set intersection in (10) requires the most resources, the prediction only required $5 \mathrm{~ms}$ for $\Delta t=0.5 \mathrm{~s}$.

Furthermore, we also validated our model using ground truth trajectories of 389 pedestrians from the publicly available BIWI Walking Pedestrians dataset of a street scene in Zurich, Switzerland [31]. Again, we achieved $100 \%$ coverage using $\Delta_{s} \in[0.15 \mathrm{~m}, 0.3 \mathrm{~m}], \Delta_{v}=0.15 \mathrm{~m} / \mathrm{s}$, and $\Delta_{\varphi} \in[0.2 \mathrm{rad}, 0.5 \mathrm{rad}]$.

\section{B. Evaluation of Rule-Based Occupancy}

Next, we demonstrate the influence of the constraints deduced from the traffic rules. As shown in Fig. 7a, the pedestrian just stepped onto the roadway (with $v_{0}=1.40 \mathrm{~m} / \mathrm{s}$ at $\left.t_{0}=0 \mathrm{~s}\right)$. According to Tab. II $b_{\text {slack }}=$ false and since $\exists t \in\left[t_{0}, t_{f}\right]:\left(\mathcal{O}_{\text {dyn }}(t) \cap \mathcal{O}_{\text {rule }}(t)\right) \ominus \mathcal{C}\left(r_{\text {ped }}\right)=\emptyset, b_{\text {stop }}=$ false The resulting occupancy $\left(\mathcal{O}_{\text {slack }} \cup \mathcal{O}_{\text {stop }}\right) \cap \mathcal{O}_{\text {dyn }}(t)$ restricts the pedestrian from completely crossing the street. For $t_{f}-t_{0}=$ $3.0 \mathrm{~s}$, the computation time was $66 \mathrm{~ms}$ for $\Delta t=0.1 \mathrm{~s}$ and was reduced by a factor of 3 for $\Delta t=0.5 \mathrm{~s}$.

When the initial state is updated at $t_{0}=0.5 \mathrm{~s}$, the pedestrian had made another step onto the roadway $\left(v_{0}=\right.$ $1.58 \mathrm{~m} / \mathrm{s}$, cf. Fig. 7b). Since the constraint management again detects that the occupancy is empty, $b_{\text {perp }}=$ false and we predict the pedestrian crossing the street perpendicular to the driving direction, as shown in Fig. 7b.

\section{Application to Evasive Motion Planning}

To demonstrate how the obtained prediction can be used for evasive trajectory planning of autonomous vehicles, we make use of a trajectory planner based on convex optimization techniques [3]. The scenarios presented next are available in the CommonRoad benchmark suite including all simulation parameters ${ }^{3}$ [34].

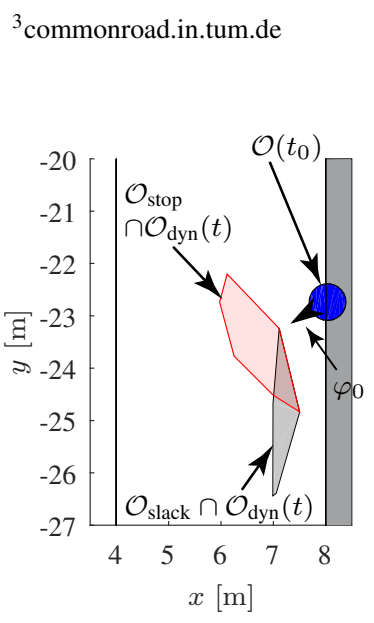

(a) $\mathcal{O}_{\text {slack }}$ and $\mathcal{O}_{\text {stop }}$ are enabled.

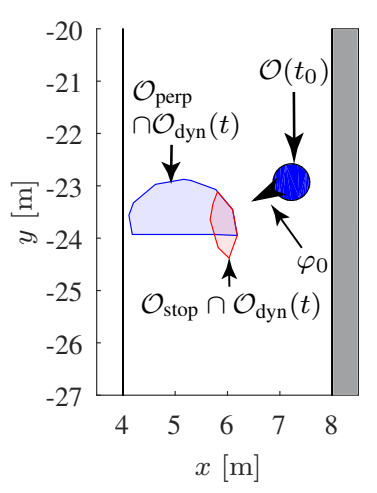

(b) $\mathcal{O}_{\text {stop }}$ and $\mathcal{O}_{\text {perp }}$ are enabled.
Fig. 7. The rule-based occupancy intersected with $\mathcal{O}_{\text {dyn }}(t)$ for different stages of a pedestrian crossing the roadway. 


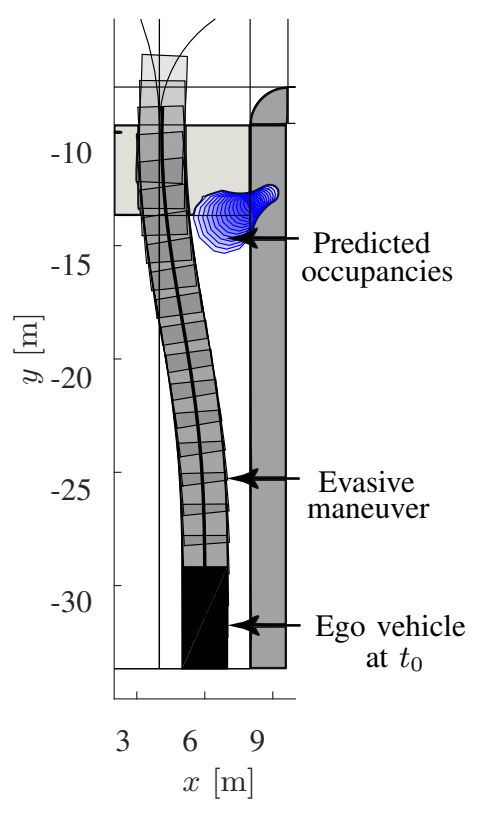

Fig. 8. By making use of our set-based prediction, planned maneuvers of the ego vehicle are guaranteed to be collision-free. As an example, we show an evasive trajectory and the predicted occupancies with $b_{\text {stop }}=$ false for $t \in[0 \mathrm{~s}, 1.8 \mathrm{~s}]$.

The ego vehicle in our first scenario (cf. Fig. 8, CommonRoad ID: S=ZAM_Intersect-1_1_S-1:2018a) is approaching the intersection with a velocity of $13.8 \mathrm{~m} / \mathrm{s}$, while the pedestrian approaches a forbidden crossing (without priority due to a red traffic light) with $v_{0}=1.35 \mathrm{~m} / \mathrm{s}$. Similar to the example in Sec. IV-B, the pedestrian cannot stop before entering the roadway and thus is predicted with $b_{\text {stop }}=$ false. We plan an evasive maneuver which involves swerving to the left adjacent lane to avoid a collision. The obtained evasive trajectory is guaranteed to be collision-free given our assumptions (cf. Fig. 8).

Furthermore, the prediction can be used to proactively evaluate evasive options so that the number of available evasive maneuvers is increased. As an example, we consider the situation $0.5 \mathrm{~s}$ later when the pedestrian has already entered the forbidden crossing $\left(v_{0}=1.40 \mathrm{~m} / \mathrm{s}\right)$, implying $b_{\text {prio }}=$ false (cf. prediction in Fig. 9. CommonRoad ID: S=ZAM_Intersect-1_2_S-1:2018a). Considering the current velocity of the ego vehicle, a collision with the crossing pedestrian can only be avoided by swerving to the left adjacent lane (similar to the maneuver of the previous scenario, cf. Fig. 8). However, this may not be an option in the presence of other vehicles. Thus, we simultaneously plan evasive trajectories for different velocities of the vehicle. As a result, we observe that the ego vehicle is still able to avoid a collision using emergency braking for a velocity of $12.5 \mathrm{~m} / \mathrm{s}$ (cf. trajectory in Fig. 9).

\section{CONCLUSIONS}

This paper proposes a formal prediction of the possible and legal future motion of pedestrians in an over-approximative, set-based fashion. By considering contextual information and the traffic rules pedestrians should adhere to, the prediction

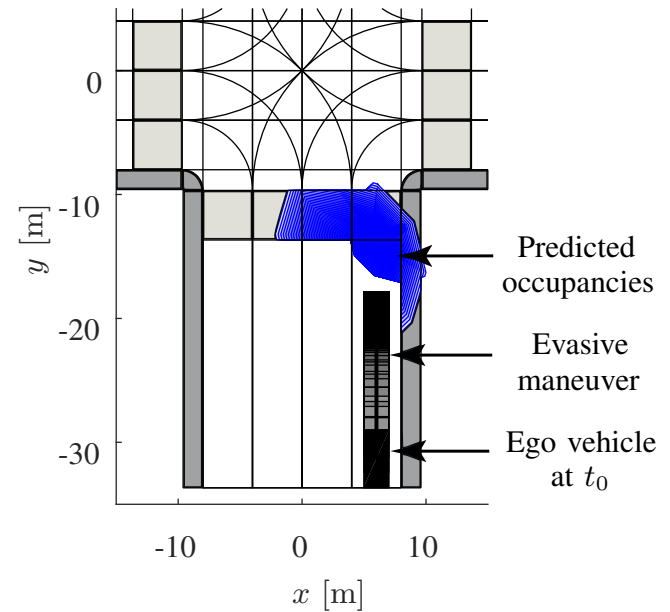

Fig. 9. The pedestrian has just entered the crossing and is predicted with $b_{\text {stop }}=b_{\text {prio }}=$ false and $t_{f}-t_{0}=5.0 \mathrm{~s}$. If the ego vehicle reduces its speed from $13.8 \mathrm{~m} / \mathrm{s}$ (in Fig. 8) to $12.5 \mathrm{~m} / \mathrm{s}$, it is also able to perform a collision-free braking maneuver.

is significantly improved compared to a solely dynamical model. Nevertheless, our approach anticipates that pedestrians disregard rules and automatically adapts the prediction to ignore violated rules. We have validated our method using recorded motions of pedestrians and highlighted its use for evasive maneuver planning.

Future work includes further studies on pedestrian behavior to validate and parameterize the constraints based on the traffic rules. Furthermore, we are currently preparing realworld vehicle experiments for evasive maneuver planning considering pedestrians crossing the road.

\section{ACKNOWLEDGMENTS}

The authors thank Sascha Steyer for the recordings of realworld data and Carmella Schürmann for the voice-over in the video attachment.

\section{REFERENCES}

[1] S. Lefèvre, D. Vasquez, and C. Laugier, "A survey on motion prediction and risk assessment for intelligent vehicles," ROBOMECH Journal, vol. 1, no. 1, pp. 1-14, 2014.

[2] M. S. Shirazi and B. T. Morris, "Looking at intersections: A survey of intersection monitoring, behavior and safety analysis of recent studies," IEEE Transactions on Intelligent Transportation Systems, vol. 18, no. 1, pp. 4-24, 2017.

[3] C. Pek and M. Althoff, "Computationally efficient fail-safe trajectory planning for self-driving vehicles using convex optimization," in Proc. of the 21th IEEE International Conference on Intelligent Transportation Systems, 2018.

[4] M. Althoff and S. Magdici, "Set-based prediction of traffic participants on arbitrary road networks," IEEE Transactions on Intelligent Vehicles, vol. 1, no. 2, pp. 187-202, 2016.

[5] F. Flohr, M. Dumitru-Guzu, J. F. P. Kooij, and D. M. Gavrila, "A probabilistic framework for joint pedestrian head and body orientation estimation," IEEE Transactions on Intelligent Transportation Systems, vol. 16, no. 4, pp. 1872-1882, 2015.

[6] A. Rasouli, I. Kotseruba, and J. K. Tsotsos, "Are they going to cross? A benchmark dataset and baseline for pedestrian crosswalk behavior," in Proc. of the IEEE International Conference on Computer Vision Workshop, 2017, pp. 206-213.

[7] A. Brunetti, D. Buongiorno, G. F. Trotta, and V. Bevilacqua, "Computer vision and deep learning techniques for pedestrian detection and tracking: A survey," Neurocomputing, vol. 300, pp. 17-33, 2018. 
[8] C. G. Keller and D. M. Gavrila, "Will the pedestrian cross? A study on pedestrian path prediction," IEEE Transactions on Intelligent Transportation Systems, vol. 15, no. 2, pp. 494-506, 2014.

[9] S. Bonnin, T. H. Weisswange, F. Kummert, and J. Schmuedderich, "Pedestrian crossing prediction using multiple context-based models,' in Proc. of the 17th IEEE International Conference on Intelligent Transportation Systems, 2014, pp. 378-385.

[10] A. T. Schulz and R. Stiefelhagen, "Pedestrian intention recognition using latent-dynamic conditional random fields," in Proc. of the IEEE Intelligent Vehicles Symposium, 2015, pp. 622-627.

[11] R. Quintero, I. Parra, J. Lorenzo, D. Fernández-Llorca, and M. A. Sotelo, "Pedestrian intention recognition by means of a hidden Markov model and body language," in Proc. of the 20th IEEE International Conference on Intelligent Transportation Systems, 2017, pp. 1-7.

[12] S. Neogi, M. Hoy, W. Chaoqun, and J. Dauwels, "Context based pedestrian intention prediction using factored latent dynamic conditional random fields," in Proc. of the IEEE Symposium Series on Computational Intelligence, 2017, pp. 1-8.

[13] J. F. P. Kooij, N. Schneider, and D. M. Gavrila, "Analysis of pedestrian dynamics from a vehicle perspective," in Proc. of the IEEE Intelligent Vehicles Symposium, 2014, pp. 1445-1450.

[14] M. Goldhammer, M. Gerhard, S. Zernetsch, K. Doll, and U. Brunsmann, "Early prediction of a pedestrian's trajectory at intersections," in Proc. of the 16th International IEEE Conference on Intelligent Transportation Systems, 2013, pp. 237-242.

[15] B. Völz, H. Mielenz, R. Siegwart, and J. Nieto, "Predicting pedestrian crossing using quantile regression forests," in Proc. of the IEEE Intelligent Vehicles Symposium, 2016, pp. 426-432.

[16] E. Rehder and H. Kloeden, "Goal-directed pedestrian prediction," in Proc. of the IEEE International Conference on Computer Vision Workshop, 2015, pp. 139-147.

[17] D. Vasquez, "Novel planning-based algorithms for human motion prediction," in Proc. of the IEEE International Conference on Robotics and Automation, 2016, pp. 3317-3322.

[18] V. Karasev, A. Ayvaci, B. Heisele, and S. Soatto, "Intent-aware long-term prediction of pedestrian motion," in Proc. of the IEEE International Conference on Robotics and Automation, 2016, pp. 2543-2549.

[19] P. Vasishta, D. Vaufreydaz, and A. Spalanzani, "Natural vision based method for predicting pedestrian behaviour in urban environments," in Proc. of the 20th IEEE International Conference on Intelligent Transportation Systems, 2017, pp. 1-6.

[20] J. Wu, J. Ruenz, and M. Althoff, "Probabilistic map-based pedestrian motion prediction taking traffic participants into consideration," in Proc. of the IEEE Intelligent Vehicles Symposium, 2018, pp. 12851292.

[21] K. C. Fuerstenberg and J. Scholz, "Reliable pedestrian protection using laserscanners," in Proc. of the IEEE Intelligent Vehicles Symposium, 2005, pp. 142-146.
[22] M. Meinecke, M. Roehder, T. Nguyen, M. Obojski, M. Heuer B. Giesler, and B. Michaelis, "Motion model estimation for pedestrians in street-crossing scenarios," in Proc. of the International Workshop on Intelligent Transportation, vol. 7, 2010.

[23] S. B. Liu, H. Roehm, C. Heinzemann, I. Lütkebohle, J. Oehlerking, and M. Althoff, "Provably safe motion of mobile robots in human environments," in Proc. of the IEEE/RSJ International Conference on Intelligent Robots and Systems, 2017, pp. 1351-1357.

[24] P. Bender, J. Ziegler, and C. Stiller, "Lanelets: Efficient map representation for autonomous driving," in Proc. of the IEEE Intelligent Vehicles Symposium, 2014, pp. 420-425.

[25] G. Lafferriere, G. J. Pappas, and S. Yovine, "A new class of decidable hybrid systems," in Hybrid Systems: Computation and Control, ser LNCS 1569. Springer, 1999, pp. 137-151.

[26] M. Althoff, "Reachability analysis and its application to the safety assessment of autonomous cars," Dissertation, Technische Universität München, 2010, http://nbnresolving.de/urn/resolver.pl?urn:nbn:de:bvb:91-diss-20100715963752-1-4.

[27] M. Althoff, C. Le Guernic, and B. H. Krogh, "Reachable set computation for uncertain time-varying linear systems," in Hybrid Systems: Computation and Control, 2011, pp. 93-102.

[28] A. Rizaldi and M. Althoff, "Formalising traffic rules for accountability of autonomous vehicles," in Proc. of the 18th IEEE International Conference on Intelligent Transportation Systems, 2015, pp. 1658 1665 .

[29] United Nations Economic Commission for Europe, "Convention on road traffic," United Nations Conference on Road Traffic, 1968, (consolidated version of 2006). [Online]. Available: https://www. unece.org/fileadmin/DAM/trans/conventn/Conv_road_traffic_EN.pdf

[30] S. Steyer, G. Tanzmeister, and D. Wollherr, "Object tracking based on evidential dynamic occupancy grids in urban environments," in Proc. of the IEEE Intelligent Vehicles Symposium, 2017, pp. 1064-1070.

[31] S. Pellegrini, A. Ess, K. Schindler, and L. van Gool, "You'll never walk alone: modeling social behavior for multi-target tracking," in Proc. of the 12th IEEE International Conference on Computer Vision, 2009, pp. 261-268.

[32] Safety of machinery - Positioning of safeguards with respect to the approach speeds of parts of the human body (ISO 13855:2010), ISO Std., 2010.

[33] N. Tiemann, "Ein Beitrag zur Situationsanalyse im vorausschauenden Fußgängerschutz," Dissertation, Universität Duisburg-Essen, 2012, https://duepublico.uni-duisburgessen.de/servlets/DerivateServlet/Derivate-31259/Tiemann_Diss.pdf.

[34] M. Althoff, M. Koschi, and S. Manzinger, "CommonRoad: Composable benchmarks for motion planning on roads," in Proc. of the IEEE Intelligent Vehicles Symposium, 2017, pp. 719-726. 